\title{
Diagnostic reliability of an immunochromatographic test for Chagas disease screening at a primary health care centre in a rural endemic area
}

\author{
Diego Mendicino ${ }^{1 /+}$, Mariana Stafuza', Carlina Colussi ${ }^{1}$, \\ Mónica del Barco', Mirtha Streiger', Edgardo Moretti² \\ ${ }^{1}$ Centre for Research on National Endemics, Faculty of Biochemistry and Biological Sciences, National University of Litoral, Santa Fe, Argentina \\ ${ }^{2}$ National Coordination for Vector Control, Faculty of Medical Sciences, National University of Cordoba, Cordoba, Argentina
}

Many patients with Chagas disease live in remote communities that lack both equipment and trained personnel to perform a diagnosis by conventional serology (CS). Thus, reliable tests suitable for use under difficult conditions are required. In this study, we evaluated the ability of personnel with and without laboratory skills to perform immunochromatographic (IC) tests to detect Chagas disease at a primary health care centre (PHCC). We examined whole blood samples from 241 patients and serum samples from 238 patients. Then, we calculated the percentage of overall agreement (POA) between the two groups of operators for the sensitivity (S), specificity (Sp) and positive $(P P V)$ and negative (NPV) predictive values of IC tests compared to CS tests. We also evaluated the level of agreement between ELISAs and indirect haemagglutination (IHA) tests. The readings of the IC test results showed 100\% agreement $(P O A=1)$. The IC test on whole blood showed the following values: $S=87.3 \% ; S p=98.8 \% ; P P V=96.9 \%$ and $N P V=95.9 \%$. Additionally, the IC test on serum displayed the following results: $S=95.7 \% ; S p=100 \%$; $P P V$ $=100 \%$ and $N P V=98.2 \%$. Using whole blood, the agreement with ELISA was $96.3 \%$ and the agreement with IHA was $94.1 \%$. Using serum, the agreement with ELISA was $97.8 \%$ and the agreement with IHA was $96.6 \%$. The IC test performance with serum samples was excellent and demonstrated its usefulness in a PHCC with minimal equipment. If the IC test $S$ value and NPV with whole blood are improved, then this test could also be used in areas lacking laboratories or specialised personnel.

Key words: Chagas disease - serological test - immunochromatographic assay

Chagas disease is a widespread zoonosis in Latin America caused by the flagellate protozoan Trypanosoma cruzi. The transmission route of Chagas disease is primarily vector borne through haematophagous triatomine insects. Triatoma infestans represents the greatest risk due to its anthropophilic habits. Other transmission routes of quantitatively lower public health significance include transfusion, connatal and digestive routes. These forms of inter-human transmission are responsible for the urbanisation and globalisation of this disease (Schmuñis \& Yadón 2010). The insect vectors of Chagas disease persist in dwellings of scattered and hard-to-reach rural populations where the prevalence of infection by this route is greater due to both environmental and social reasons (Briceño-León \& Galván 2007).

T. cruzi infection is often asymptomatic or oligosymptomatic during the acute period. The chronic stage of infection is also latent until after 20 years or more when cardiac (most common) and/or gastrointestinal pathologies appear in approximately one-third of patients.

doi: $10.1590 / 0074-0276140153$

Financial support: $\mathrm{CAI}+\mathrm{D} / \mathrm{UNL}$

+ Corresponding author: diegomendicino@hotmail.com

Received 4 May 2014

Accepted 6 November 2014
T. cruzi infection is confirmed during the chronic phase by demonstrating the host immune response against this parasite. At least two standardised serological tests with different principles or that detect antibodies against different specific antigens must be used for confirmation (Rassi Jr et al. 2010). To diagnose Chagas disease, the results of the two tests must be coincidentally positive. In cases of discrepancy (a positive and a negative test), a third test should be conducted to confirm or rule out infection.

The most commonly used serological tests [conventional serology (CS)] are ELISAs and indirect haemagglutination (IHA) tests. Currently, various serological diagnostic techniques are being evaluated, with each technique using different detection principles (Afonso et al. 2012). However, conducting these tests requires equipped laboratories and human resources trained in biochemical analysis.

Vector transmission occurs most often in rural populations far from urban centres. In these areas, access to health coverage is reduced and travelling to locations where more complex services are available is extremely difficult.

In some provinces in developing countries, births commonly occur without any medical care. Therefore, many children do not have access to laboratory testing for the early detection of congenital transmission. Additionally, in unexpected situations in which an emergency transfusion is required (e.g., accidents, natural disasters and weapon injuries), people must choose between the 
risk involved in the delay caused by the transfer of the patient and the risk of using blood that has not been screened for Chagas.

Some authors have proposed collecting blood samples on filter paper (Holguín et al. 2013) or storing blood samples in glycerin (Arrieta et al. 2004) for clinical and population studies in rural areas due to the lack of availability of laboratories with the necessary equipment to diagnose this infection in these areas. The analysis of biological samples obtained by non-invasive methods, such as oral mucosal transudate specimens, has also been suggested (Moretti et al. 2004). Invariably, the samples must subsequently be taken to a laboratory for processing. However, this approach requires revisiting patients with positive results to confirm the infection using a newly collected biological sample.

Because of these difficulties, techniques that can be used in primary health care centres (PHCCs) without highly complex requirements, such as specialised personnel and instruments, are required. However, such techniques must be able to deliver results quickly and reliably.

An immunochromatographic (IC) test is a rapid visually read test that is widely used for the diagnosis of physiological conditions (e.g., pregnancy tests) and of infectious and non-infectious diseases. Several IC tests can be performed on whole blood. The reagents for these tests are stored at room temperature (RT); thus, these tests do not require cold storage and the data can be easily acquired and interpreted. These characteristics will make new techniques suitable for use in low-resource settings (López-Chejade et al. 2010). For use as screening techniques, assays must also have extremely high sensitivity (S) and negative predictive values (NPV) because excluding patients who seem to be seronegative, but who are actually positive is problematic. Positive samples should be sent to more complex laboratories for confirmation by other techniques. The techniques should have high specificity (Sp) and positive predictive values (PPV) to avoid excessive labour use and economic burdens on the health care system. Incorrect test results also cause an emotional burden on the patients and their families.

We compared the results obtained with this test in the field using whole blood and serum samples with results from CS to study the performance and the usefulness of a commercial IC test for the diagnosis of Chagas infection. The results of the IC tests were read by staff both with and without training in laboratory work.

\section{SUBJECTS, MATERIALS AND METHODS}

Patient selection and sample collection - Trained laboratory technicians obtained blood samples by venipuncture using disposable syringes and needles from 241 patients attending clinical PHCC in the rural area of the province of Santa Fe, Argentina. The prevalence and risk of Chagas in the study area are similar to those found in the Gran Chaco ecoregion, although the vector situation is under control in Santa Fe (Mendicino et al. 2013). The study was "single-blind" because the tested samples were obtained from a serological survey of patients at a greater risk of being infected due to their epidemiological history (unsafe dwelling conditions and/or a mother with Chagas disease). The single-blind design was chosen because of the low prevalence of this disease and because a double-blind design would have required an excessively high number of samples.

The samples were collected in disposable tubes with separator gel. No plasma samples were used so that the samples could be transported in the primary tubes without haemolysis.

Processing the whole blood IC test in the field - The whole blood samples were tested using WL Check Chagas test kits (Wiener Lab SAIC, Argentina). The WL Check Chagas test is a lateral flow IC test that detects antibodies specific to T. cruzi. These kits do not include any disposable items (e.g., capillary tubes or Pasteur pipettes) and the only laboratory instrument required to perform this test is an automatic micropipette. Each test kit consists of individual tests that can be stored at RT; the test results should be read after 25-35 min. Each individual cassette has an internal quality control. If the sample contains antibodies specific to $T$. cruzi, then the complex bound to the antigens on the membrane produces a pink and/or purple line. If the sample does not contain antibodies to T. cruzi, then the complex remains unbound and no line appears.

To conduct the IC tests, we collected $40 \mu \mathrm{L}$ of whole blood with an automatic micropipette before a clot formed and then followed the manufacturer's instructions for the IC test. After the required waiting time, the results were read in situ by two different operators, who were a qualified health professional (biochemist, lab technician, nurse or doctor) and an unqualified volunteer (driver or administrative staff). The results were recorded in spreadsheets in a single-blind manner.

The sera were separated by centrifugation at the PHCC. The samples were transported to the reference hospital in their primary tubes and stored in compliance with the corresponding biosafety and conservation regulations.

Processing serum IC and CS samples in the laboratory - All samples were subjected to ELISA (Chagatest ELISA, Wiener Lab SAIC) and IHA (IHA Chagas Polychaco, Lemos Laboratory SRL, Argentina) in the laboratory of the Centre for Research on National Endemic Diseases of the National University of Litoral (UNL) using commercial reagents approved by the National Administration of Drugs, Food and Medical Technology. When a discrepancy between the two tests occurred, we performed indirect immunofluorescence with commercial conjugates and smears were prepared in our laboratory with formalin-fixed T. cruzi epimastigotes of the Tulahuen strain (Streiger et al. 1980). A sample was considered positive or negative according to the agreement of two of these three CS tests. The CS results were delivered to the individual patients.

Serum could not be obtained from three of the 241 patients because the volume after centrifugation was too small. IC tests were performed on 238 serum samples from the same patients following the manufacturer's instructions.

Data analysis - The results were entered into a database in Microsoft Excel and analysed using Epi Info 3.5.1 software. 
TABLE I

Results of immunochromatography (IC) with whole blood vs conventional serology (CS)

\begin{tabular}{llccc}
\hline & & \multicolumn{2}{c}{ CS } & \\
\cline { 3 - 4 } & & Positive & Negative & Total \\
\hline \multirow{2}{*}{ IC on whole blood } & Positive & 62 & 2 & 64 \\
& Negative & 9 & 168 & 177 \\
\cline { 2 - 4 } & Total & 71 & 170 & 241 \\
\hline
\end{tabular}

negative predictive value: $95.9 \%$; positive predictive value: 96.9\%; sensitivity: $87.3 \%$; specificity: $98.8 \%$.

The percentage of overall agreement (POA) was determined as a measure of the variation in readings between observers with $(\mathrm{O} 1)$ and observers without $(\mathrm{O} 2)$ training in laboratory work. The POA was calculated as $\mathrm{a}+\mathrm{d} / \mathrm{a}+\mathrm{b}+\mathrm{c}+\mathrm{d}$, where $\mathrm{a}=$ positive readings for $\mathrm{O} 1$ and $\mathrm{O} 2, \mathrm{~b}=$ a positive reading for $\mathrm{O} 1$ and a negative reading for $\mathrm{O} 2, \mathrm{c}=$ a negative reading for $\mathrm{O} 1$ and a positive reading for $\mathrm{O} 2$ and $\mathrm{d}=$ negative readings for $\mathrm{O} 1$ and $\mathrm{O} 2$.

We calculated the S, Sp, PPV and NPV by comparing the IC test results on whole blood and serum with the CS results. We considered the results as true positive (TP) when the IC and CS were positive for the sample and true negative (TN) when the IC and CS results were negative. A false positive (FP) was defined as a positive IC result and a negative CS result. A false negative (FN) was defined as a negative IC result and a positive CS result.

$\mathrm{S}$ is defined as the proportion of study participants considered positive by CS who were correctly identified as positive by IC test. Sp is defined as the proportion of study participants without Chagas infection according to CS who were correctly identified by IC test. We calculated $\mathrm{S}$ and $\mathrm{Sp}$ using the following equations: $\mathrm{S}=\mathrm{TP} /$ $\mathrm{TP}+\mathrm{FN}$ and $\mathrm{Sp}=\mathrm{TN} / \mathrm{TN}+\mathrm{FP}$.

PPV is defined as the proportion of study participants who were reactive to the IC test (TP+FP) and who were correctly considered positive by the IC test (TP). Therefore, PPV represents the percentage of patients who actually had the disease and who also tested positive. The NPV is defined as the proportion of patients who were non-reactive for this test $(\mathrm{TN}+\mathrm{FN})$ and who were correctly considered negative by the IC test (TN). The NPV represents the percentage of patients without the disease who also tested negative. We calculated these values using the following equations: $\mathrm{PPV}=\mathrm{TP} / \mathrm{FP}+\mathrm{TP}$ and $\mathrm{NPV}=\mathrm{TN} / \mathrm{FN}+\mathrm{TN}$.

We also determined the agreement of the IC results with the IHA and ELISA results. This agreement was defined as the proportion of the IC results (positive and negative) that coincided with the IHA and ELISA results.

Ethics - This study was reviewed and approved by the Advisory Committee on Research Ethics and Safety of the Faculty of Biochemistry and Biological Sciences of the UNL.
TABLE II

Results of immunochromatography (IC)

on serum vs conventional serology (CS)

\begin{tabular}{llccc}
\hline & & \multicolumn{3}{c}{ CS } \\
\cline { 3 - 4 } & & Positive & Negative & Total \\
\hline \multirow{2}{*}{ IC on serum } & Positive & 67 & 0 & 67 \\
& Negative & 3 & 168 & 171 \\
\cline { 2 - 4 } & Total & 70 & 168 & 238 \\
\hline
\end{tabular}

negative predictive value: $98.2 \%$; positive predictive value: 100\%; sensitivity: $95.7 \%$; specificity: $100 \%$.

Before collecting the blood samples, the scope of the study was explained to the patients and all questions were addressed. We included all patients who accepted being subjected to analysis for Chagas by signing an informed consent form. The CS results were given to each patient individually and privately. The seropositive patients were connected with the provincial program for Chagas control, monitoring and treatment in accordance with the National Standards for Care.

\section{RESULTS}

Variation in the readings between observers - The agreement $(\mathrm{POA}=1)$ between the readings of the results of the IC tests on whole blood was $100 \%$ when assessed by different operators (personnel with and without training in laboratory work).

$S, S p$ and PPV and NPV - As shown in Table I, the results of the IC tests on whole blood had an $\mathrm{S}$ of $87.3 \%$ and an Sp of $98.8 \%$ compared with CS. The PPV was $96.9 \%$ and the NPV was $95.9 \%$.

When the results of the IC tests on serum samples were compared with the results of CS, S was $95.7 \%$, Sp was $100 \%$, PPV was $100 \%$ and NPV $98.2 \%$ (Table II).

Agreement with ELISA and IHA - A comparison of the results of the IC tests on whole blood and the results of the ELISA and IHA tests are presented in Table III. The result agreement was $96.3 \%$ with ELISA and $94.1 \%$ with IHA.

The results of the IC tests on serum and the results of the ELISA and IHA tests are summarised in Table IV. The agreement between the IC and ELISA results was $97.8 \%$ and that between the IC and IHA results was $96.6 \%$.

\section{DISCUSSION}

IC tests are advantageous for the diagnosis of Chagas disease in low-resource settings such as rural areas because these tests are easy to use and can be performed with both whole blood and serum. Despite these advantages, only a limited number of studies regarding the use of these tests with whole blood in the field have been published (Roddy et al. 2008, Chippaux et al. 2009). Several studies have evaluated the performance of these tests with serum samples in the laboratory (Ponce et al. 2005, Reithinger et al. 2010). In a recent multicentre 
TABLE III

Results of immunochromatography (IC) on whole blood vs ELISA and indirect haemagglutination (IHA)

\begin{tabular}{|c|c|c|c|c|c|c|}
\hline & & \multicolumn{2}{|c|}{ ELISA } & \multicolumn{2}{|c|}{ IHA } & \multirow[b]{2}{*}{ Total } \\
\hline & & Positive & Negative & Positive & Negative & \\
\hline \multirow{3}{*}{ IC on whole blood } & Positive & 62 & 2 & 62 & 2 & 64 \\
\hline & Negative & 77 & 170 & 12 & 165 & 177 \\
\hline & Total & 69 & 172 & 73 & 165 & 241 \\
\hline
\end{tabular}

agreement with ELISA: 96.3\% [(62+170)/241]; agreement with IHA: 94.1\% [(62+165)/241].

TABLE IV

Results of immunochromatography (IC) on serum vs ELISA and indirect haemagglutination (IHA)

\begin{tabular}{|c|c|c|c|c|c|c|}
\hline & & \multicolumn{2}{|c|}{ ELISA } & \multicolumn{2}{|c|}{ IHA } & \multirow[b]{2}{*}{ Total } \\
\hline & & Positive & Negative & Positive & Negative & \\
\hline \multirow[t]{3}{*}{ IC on serum } & Positive & 65 & 2 & 66 & 1 & 67 \\
\hline & Negative & 3 & 168 & 7 & 164 & 171 \\
\hline & Total & 68 & 170 & 73 & 165 & 238 \\
\hline
\end{tabular}

agreement with ELISA: 97.8\% [(65+168)/238]; agreement with IHA: 96.6\% [(66+164)/238].

study, the performances of 11 commercially available IC tests for Chagas disease (including the WL Check Chagas test) were compared using samples from reference laboratory serum banks (Sánchez-Camargo et al. 2014). That comparison was performed under laboratory conditions and the authors concluded that further studies should be conducted in either the field or clinical settings in both endemic and non-endemic areas using whole blood samples. The present study was performed in a low-resource setting with serum and whole-blood samples and the results were read by trained and untrained personnel. The behaviour of the tests has not been previously compared using both types of samples or using the variability in readings obtained from trained and untrained personnel.

The IC test evaluated in this study was easy to implement. Although this test was performed by personnel untrained in laboratory work, their readings agreed 100\% with those made by health personnel trained in conducting laboratory tests. Sánchez-Camargo et al. (2014) also reported a high score in the ease-of-use questionnaire for the same test when performed under laboratory conditions. A previous study with another commercial IC test showed $99.9 \%$ agreement among two non-laboratory health workers (Roddy et al. 2008). Although diagnostic tests should be performed by laboratory personnel, these tests can be performed in places where health workers are scarce or during acute emergencies, such as natural disasters, when emergency transfusions are required and when blood banks are not accessible.
A high level of agreement was observed among the results obtained in the laboratory with serum samples using IC, ELISA and IHA tests. The use of ELISA and IHA is widespread in biochemical diagnosis centres. However, IHA has a lower S and a lower percentage of agreement compared with the other tests (Remesar et al. 2009).

The values of S, Sp, PPV and NPV were acceptable when the results of the tests with serum were compared with those of the CS results and were similar to the results obtained with the same commercial test in the study led by Sánchez-Camargo et al. (2014). The performances of IC tests in the laboratory were similar to those of conventional techniques (Remesar et al. 2009, Flores-Chávez et al. 2010, Afonso et al. 2012, Pereira et al. 2012), with the advantage that IC tests require only a centrifuge to separate the serum.

The results obtained with the kit used in this study and whole blood samples showed $\mathrm{S}$ values lower than those obtained with other IC tests for Chagas in both field (Roddy et al. 2008, Chippaux et al. 2009) and laboratory studies (Ponce et al. 2005). The Sp value was similar to the $\mathrm{Sp}$ values observed in those studies. The agreement with validated techniques (ELISA and IHA) was lower than when using serum samples.

The performance of the test evaluated in the present study is acceptable for use in screening American trypanosomiasis if performed with serum and confirmed with other reference techniques. This test requires only basic equipment to separate the serum, the performance 
and interpretation of the test are simple and the reading time is short. Because of these characteristics, this test is suitable for use in both low-complexity laboratories and PHCCs that are often more accessible to populations in vector risk areas.

Each cassette of the kit has an internal quality control so that the determinations can be made individually without processing a batch of samples from different patients. Individual determination is one advantage of this test over IHA and ELISA, which both require positive and negative controls with each processing batch. Thus, IC tests are convenient for use in laboratories in which an analysis for Chagas is not required frequently.

When performed with whole blood samples, we believe that this IC test does not have the necessary $\mathrm{S}$ and NPV to be used as a screening technique in low-resource settings and would lead to incorrectly identifying some patients as non-infected. If these parameters are improved, then this test could be useful for diagnosis in the field and in population studies. This test could also be useful in pre-transfusion screening in emergencies and in situations with no health facilities or with health facilities with limited access to the population.

Evaluating the performance of the IC test on alternative samples, such as oral mucosal transudate specimens, which are collected using a non-invasive method and are advantageous in terms of biosafety and acceptance by the study population, will also be valuable.

\section{ACKNOWLEDGEMENTS}

To the communities that worked with us and to the staff of the PHCC, for allowing us to conduct the study, and to the Provincial Chagas Program of the Province of Santa Fe and its director, Marcelo Nepote, for logistical support in the field trips.

\section{REFERENCES}

Afonso AM, Ebell MH, Tarleton RL 2012. A systematic review of high quality diagnostic tests for Chagas disease. PLoS Negl Trop Dis 6: e1881.

Arrieta R, Daquino B, Rosso N, Ferreras M, Juárez N 2004. Evaluación de una metodología de tamizaje en la enfermedad de Chagas en San Luis, Argentina. Salud Publica Mex 46: 430-437.

Briceño-León R, Galván JM 2007. The social determinants of Chagas disease and the transformations of Latin America. Mem Inst Oswaldo Cruz 102 (Suppl. I): 109-112.

Chippaux J, Santalla J, Postigo J, Romero M, Clavijo NS, Schneider D, Brutus L 2009. Sensitivity and specificity of Chagas Stat-Pak test in Bolivia. Trop Med Int Health 14: 732-735.

Flores-Chávez M, Cruz I, Rodríguez M, Nieto J, Franco E, Gárate T, Cañavate C 2010. Comparación de técnicas serológicas convencionales y no convencionales para el diagnóstico de la enfermedad de Chagas importada en España. Enferm Infecc Microbiol Clin 28: 284-293.
Holguín A, Norman F, Martín L, Mateos M, Chacón J, López-Vélez R, Pérez-Molina J 2013. Dried blood as an alternative to plasma or serum for Trypanosoma cruzi IgG detection in screening programs. Clin Vaccine Immunol 20: 1197-1202.

López-Chejade P, Roca C, Posada E, Pinazo M, Gascon J, Portús M 2010. Utilidad de un test inmunocromatográfico para el cribado de la enfermedad de Chagas en asistencia primaria. Enferm Infecc Microbiol Clin 28: 169-171.

Mendicino D, Stafuza M, del Barco M, Colussi C, Bizai M, Fabbro D, Nepote M, Streiger M 2013. Infección chagásica en niños de cuatro distritos de riesgo de la provincia de Santa Fe. Acta Bioquím Clín Latinoam 47: 571-578.

Moretti E, Basso B, Gil P, Vaca B, Jacqueline J, Yasenzaniro P 2004. Detección de anticuerpos para Chagas y toxoplasmosis en trasudado mucoso oral. Acta Bioquím Clín Latinoam 38: 159-163.

Pereira GA, Lozada-Neto F, Barbosa VF, Ferreira-Silva M, de Moraes-Souza H 2012. Performance of six diagnostic tests to screen for Chagas disease in blood banks and prevalence of Trypanosoma cruzi infection among donors with inconclusive serology screening based on the analysis of epidemiological variables. Rev Bras Hematol Hemoter 34: 292-297.

Ponce C, Ponce E, Vinelli E, Montoya A, de Aguilar V, González A, Zingales B, Rangel-Aldao R, Levin M, Esfandiari J, Umesawa E, Luquetti A, da Silveira J 2005. Validation of a rapid and reliable test for diagnosis of Chagas disease by detection of Trypanosoma cruzi-specific antibodies in blood donors and patients in Central America. J Clin Microbiol 43: 5065-5068.

Rassi Jr A, Rassi A, Marin-Neto J 2010. Chagas disease. Lancet 375: 1388-1402.

Reithinger R, Grijalva M, Chiriboga R, de Noya BA, Torres J, Pavia-Ruz N, Manrique-Saide P, Cardinal M, Gürtler R 2010. Rapid detection of Trypanosoma cruzi in human serum by use of an immunochromatographic Dipstick Test. J Clin Microbiol 48: 3003-3007.

Remesar M, Gamba C, Colaianni I, Puppo M, Sartor P, Murphy E, Neilands T, Ridolfi M, Leguizamón M, Kuperman S, del Pozo A 2009. Estimation of sensitivity and specificity of several Trypanosoma cruzi antibody assays in blood donors in Argentina. Transfusion 49: 2352-2358.

Roddy P, Goiri J, Flevaud L, Palma P, Morote S, Lima N, Villa L, Torrico F, Albajar-Viñas P 2008. Field evaluation of a rapid immunochromatographic assay for detection of Trypanosoma cruzi infection by use of whole blood. J Clin Microbiol 46: 2022-2027.

Sánchez-Camargo C, Albajar-Viñas P, Wilkins P, Nieto J, Leiby D, Paris L, Scollo K, Flórez C, Guzmán-Bracho C, Luquetti A, Calvo N, Tadokoro K, Saez-Alquezar A, Palma P, Martin M, Flevaud L 2014. Comparative evaluation of 11 commercialized diagnostic tests for detecting Trypanosoma cruzi antibodies in serum banks in endemic and non-endemic areas. $J$ Clin Microbiol doi: 10.1128/JCM.00144-14.

Schmuñis G, Yadón Z 2010. Chagas disease: a Latin American health problem becoming a world health problem. Acta Trop 115: 14-21.

Streiger ML, Bovero NM, Dávila EV 1980. Reacción de inmunofluorescencia indirecta para el diagnóstico de la infección chagásica. Conservación de improntas. Medicina (B Aires) 40: 250-251. 